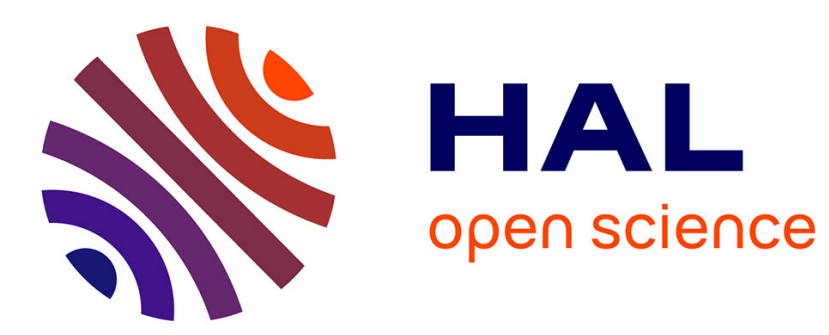

\title{
Fatigue thermique des aciers à outils pour travail à chaud
}

Sylvain Jean, Jean-Paul Arcens, Serge Tovar, Farhad Rezai-Aria

\section{To cite this version:}

Sylvain Jean, Jean-Paul Arcens, Serge Tovar, Farhad Rezai-Aria. Fatigue thermique des aciers à outils pour travail à chaud. Matériaux \& Techniques, 1999, nº1-2, p. 23-29. 10.1051/mattech/199987010023 . hal-01851451

\section{HAL Id: hal-01851451 \\ https://hal.science/hal-01851451}

Submitted on 6 Nov 2019

HAL is a multi-disciplinary open access archive for the deposit and dissemination of scientific research documents, whether they are published or not. The documents may come from teaching and research institutions in France or abroad, or from public or private research centers.
L'archive ouverte pluridisciplinaire HAL, est destinée au dépôt et à la diffusion de documents scientifiques de niveau recherche, publiés ou non, émanant des établissements d'enseignement et de recherche français ou étrangers, des laboratoires publics ou privés. 


\title{
FATIGUE THERMIQUE DES ACIERS À OUTILS POUR TRAVAIL Ã CHAUD
}

\author{
S. Jean, J.-P. Arcens, S. Tovar et F. Rézaï-Aria \\ École des Mines Albi-Carmaux, Campus Jarlard, Albi
}

THERMAL FATIGUE OF HOT WORK TOOL STEELS

ABSTRACT: Thermal fatigue of X $38 \mathrm{CrMoV} 5$ (formerly Z38CDV5), a $5 \% \mathrm{Cr}$ steel, is investigated, in as quenched and tempered ( $47 \mathrm{HRC})$ as well as annealed conditions. A thermal fatigue rig using high frequency induction heating is developed. Tubular specimens are used. By modifying the specimen geometry, various thermal gradients and therefore different thermo-mechanical loading i.e. mechanical strain versus temperature loops are generated.

Finite element calculations of the thermo-mechanical strains and stresses reveal that the stress state in the centre of the external surface of the thermal fatigue specimen is quasi bi-axial and does not change for the different geometries used. Outside of this region, the stress ratio (i.e. hoop stress $\left(\sigma_{\theta \theta}\right)$ over axial stress $\left(\sigma_{z z}\right)$ ) arises progressively to about 2 to 2.5 (uni-axial condition).

Depending upon the maximum temperature of the thermal cycle and the amplitude of the mechanical strain generated by the thermal gradient, bi-axial oxide-scale spalling or heat checking were observed. Heat checking (bi-axial cracking) was predominantly observed in the centre of the specimens while towards to the ends of the specimens, the uni-axial cracking proceeds.

Microhardness measurements at room temperature reveal a thermal fatigue softening in as quenched and tempered steel (47 HRC). A higher maximum temperature of the thermal cycle and a higher mechanical strain increases thermal fatigue softening. The role of the number of the thermal cycles was overshadowed by the more important effect of the amplitude of the mechanical strain and the temperature. A quasi linear softening is observed over few millimetres beneath the external surface. The hardness achieve then the initial hardness of the steel. The softening rate i.e. hardness over the thermo-mechanically affected zone width is controlled by the thermal gradient and thus the thermo-mechanical loading. No softening was observed in annealed steel.

\section{Introduction}

$\mathrm{D}$ e nombreuses pièces industrielles fonctionnant à température élevée subissent des sollicitations thermiques et mécaniques lors de leur fonctionnement [1-9]. Par exemple, à chaque opération de fabrication d'une nouvelle pièce par forgeage, matriçage ou estampage à chaud, des variations plus ou moins rapides de température se produisent à la surface de travail de l'outil en raison de l'échange de chaleur entre le lopin chaud et la surface de contact de l'outillage [10]. Par conduction de la chaleur à l'intérieur de l'outillage, des vagues de gradients thermiques transitoires se développent dans l'outil. Lors de l'éjection de la pièce fabriquée, la surface de l'outillage peut se refroidir plus rapidement que les éléments internes. Ainsi, la direction de diffusion de la chaleur s'inverse car les éléments internes se trouvent à des températures plus élevées que ceux de la surface de travail. Le refroidissement de l'outillage peut être accéléré par la pulvérisation d'un lubrifiant liquide sur l'outillage et aussi par des circuits de refroidissement internes.

Conférence présentée au colloque sur les "Aciers pour moules et outils" organisé les 27 et 28 mai 1998 à l'Ecole des Mines d'Albi par le Cercle d'Etude des Métaux. A chaque instant d'un cycle thermique, la dilatation ou la contraction de chaque élément d'un outil peut être restreinte par d'autres éléments qui se trouvent à des températures différentes. En général, chaque élément est soumis à un chargement multiaxial 
mais, afin de simplifier le problème, nous examinons le cas d'une sollicitation uniaxiale. De la différence entre la dilatation libre, définie comme $\varepsilon_{t h}=\alpha \times \Delta T$ ( $\alpha$ étant le coefficient de dilatation thermique, et $\Delta T$ étant la différence entre une température donnée $T$ et une température de référence, par exemple $\left.20^{\circ} \mathrm{C}\right)$, et la dilatation restreinte $\left(\varepsilon_{\text {rest }}\right)$ d'un élément donné, il en résulte une déformation appelée "mécanique" $\left(\varepsilon_{m}=\varepsilon_{\text {rest }}-\varepsilon_{t h}\right)$ [9]. Quand la dilatation libre d'un élément est restreinte par d'autres éléments, la déformation mécanique $\left(\varepsilon_{m}\right)$ devient une déformation en compression pendant que la température de l'élément s'accroît. Dépendant de la sévérité du gradient thermique, cet élément peut être mis en traction pendant son refroidissement. Le trajet température-déformation mécanique que subit un élément est appelé "cycle" ou "histoire" thermomécanique.

Cependant, pour mettre les pièces en forme, des outillages subissent, de plus, des efforts purement mécaniques. Ces forces s'additionnent aux efforts cycliques d'origine purement thermique et c'est l'ensemble de ces deux efforts qui provoque des sollicitations thermo-mécaniques complexes de l'outillage. Chaque élément d'un outil est donc sollicité en fatigue anisotherme de type généralement compression/traction avec un cycle thermo-mécanique différent des autres éléments.

La sollicitation thermique et mécanique cyclique peut altérer les caractéristiques mécaniques en sous-surface de contact et aussi en volume des outillages (vieillissement, précipitations, transformation de phases, fragilisation, abaissement de la ténacité, etc.). La perte de la résistance mécanique des outillages est assez souvent identifiée par la mesure de dureté à froid et elle est expliquée par la thermique des outillages (surchauffe), alors que la sollicitation mécanique cyclique peut aussi largement contribuer à la perte de résistance mécanique [11-12].

Par la répétition du cycle compression/tension, le matériau s'endommage progressivement par le processus de la fatigue anisotherme. Des fissures s'amorcent et se propagent dans l'outillage à partir de la surface de contact (site privilégié pour l'amorçage de fissure). La durée de vie à l'amorçage et la vitesse de propagation de fissure sont contrôlées par la sévérité des cycles de fatigue thermo-mécanique que subit l'outillage. L'amplitude de la déformation mécanique à la surface de travail est contrôlée par la sévérité du gradient thermique dans l'outillage, les efforts de mise en forme de pièce et par le comportement de l'acier ou du système de matériaux utilisés (acier et couche nitrurée ou rechargée, par exemple).

Toutefois, le périssement des outillages n'a pas uniquement une origine purement thermo-mécanique, mais bien souvent il est le résultat d'une sollicitation "thermo-physico-chimique" à cause de l'interaction complexe de la sollicitation thermomécanique et d'un environnement oxydant ou corrosif.

\section{Approches techniques et scientifiques}

L'endommagement des aciers à outils sous sollicitations thermo-mécaniques peut être étudié par deux approches scientifiques et techniques complémentaires:

\section{Fatigue thermique}

En général, une éprouvette est chauffée et refroidie sans contrainte externe. Comme dans un outil en service, des chocs thermiques plus au moins sévères peuvent être obtenus. Deux types de paramètres contrôlent le comportement et l'endomma- gement et donc la durée de vie des matériaux en fatigue thermique: les paramètres extrinsèques, à savoir les caractéristiques de cyclage thermique, et les paramètres intrinsèques, à savoir les propriétés thermo-physico-chimiques et mécaniques des matériaux.

Les paramètres extrinsèques (conditions de sollicitation) peuvent être modifiés soit en changeant les caractéristiques du cycle thermique (température maximum et minimum, fréquence de chauffage et de refroidissement, etc.) pour une géométrie d'éprouvette donnée, soit en modifiant la géométrie d'éprouvette et en maintenant les mêmes caractéristiques du cycle thermique [1-3]. Les paramètres intrinsèques sont liés au choix des matériaux et dépendent fortement donc des propriétés physicochimiques et mécaniques du matériau examiné (le coefficient de dilatation thermique, la conductivité thermique, la taille de grain, l'orientation cristallographique, le module d'élasticité, la limite d'élasticité, etc.).

Cependant, les sollicitations thermiques et mécaniques ne sont pas directement accessibles et doivent être calculées par des analyses thermo-mécaniques appropriées [2, 13-14]. Des lois de comportement sont donc nécessaires. La performance de ces lois doit être examinée dans les conditions de sollicitation isotherme (fluage, fatigue, relaxation de contrainte, etc.) et de sollicitation anisotherme (fatigue thermo-mécanique).

\section{Fatigue thermo-mécanique}

Une éprouvette de fatigue subit un cycle de température-déformation mécanique répété de sorte que le gradient thermique dans sa partie utile soit quasi nul ou acceptable [15-17]. Ainsi, l'histoire "température-déformation mécanique-contrainte" d'un élément d'un outillage (à la surface de travail par exemple) ou d'une éprouvette de fatigue thermique peut être approchée par des mesures directes de ces grandeurs. Le cycle hypothétique de température-déformation mécanique de la surface de travail d'un outillage est schématiquement présenté sur la figure 1. Dans ce cycle, dit "cycle hors-phase", l'éprouvette subit une déformation en compression lors de l'élévation de la température. Généralement, la déformation mécanique minimale coïncide avec la température maximale du cycle thermique $\left(T_{\max }\right)$.

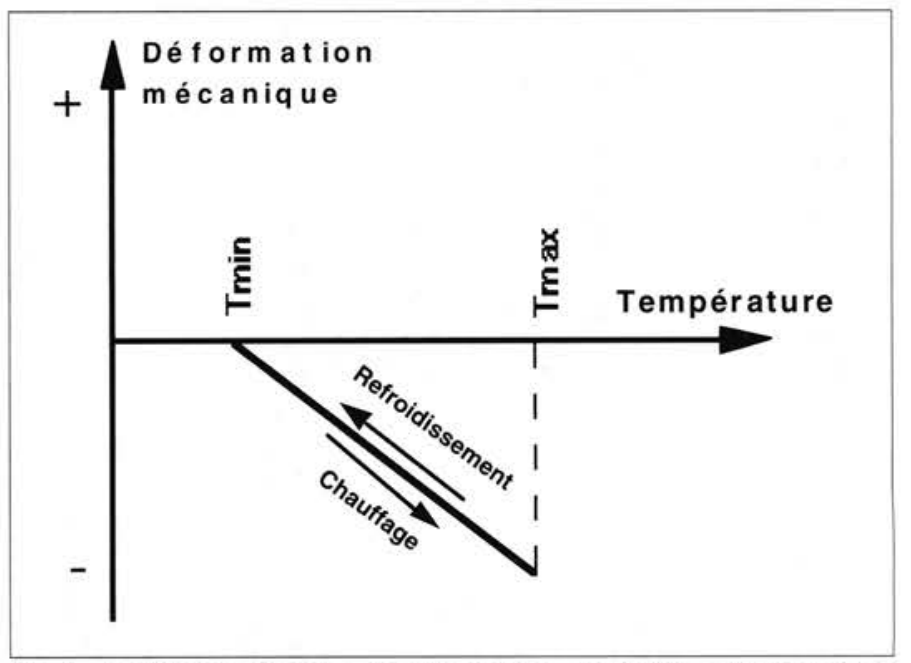

Fig. 1 - Sollicitation thermo-mécanique typique d'un élément de la surface de travail d'un outillage travaillant à chaud.

Fig. I - Typical thermo-mechanical cycle of a hot work tool for an element of the contact surface. 


\section{Objectif}

Cet article décrit le principe d'un banc d'essai de fatigue thermique (première approche) développé à l'École des Mines d'Albi-Carmaux dans le cadre de l'ACR-II (Action Concertée de Recherche en Forge) pour étudier et modéliser le comportement et l'endommagement anisothermes des aciers martensitiques. Le principe du banc est expliqué. L'effet de la température maximum, de l'amplitude de déformation mécanique et du gradient thermique sur la perte de résistance et les mécanismes d'endommagement sont discutés. Le principe de deuxième approche est reporté ailleurs [17].

\section{Banc d'essai de fatigue thermique}

L'essai de fatigue thermique est conduit sur des éprouvettes avec deux types de géométries, cylindriques et creuses (paramètres extrinsèques), représentées figure 2. Ces éprouvettes permettent de simuler et d'étudier l'effet d'un refroidissement interne (dans un outil réel par exemple) et donc la sévérité du gradient thermique sur l'endommagement des matériaux. Les éprouvettes sont chauffées par induction avec un générateur à haute fréquence ( 100 à $300 \mathrm{kHz}$ ) de $25 \mathrm{~kW}$ (CELES). L'intérieur des éprouvettes est en permanence refroidi par eau. La surface externe des éprouvettes peut être refroidie par le soufflage d'air comprimé. Les éprouvettes sont tenues par deux porte-échantillons qui se vissent sur leurs extrémités. Les deux porteéchantillons reposent sur des vis métalliques permettant de positionner et de centrer d'une manière précise les éprouvettes par rapport à l'axe de la bobine d'induction.

Dans l'optique de la mesure de déformation longitudinale [9], une spire spéciale a été développée [18]. Le centrage de l'éprouvette par rapport à la spire est essentiel pour réduire au minimum les gradients longitudinaux et circonférenciels sur la surface externe. Ainsi, seul l'effet du gradient thermique radial dans l'éprouvette est examiné. Étant donné que les éprouvettes ne sont pas encastrées pendant le chauffage et le refroidissement, elles peuvent se dilater et se contracter librement.
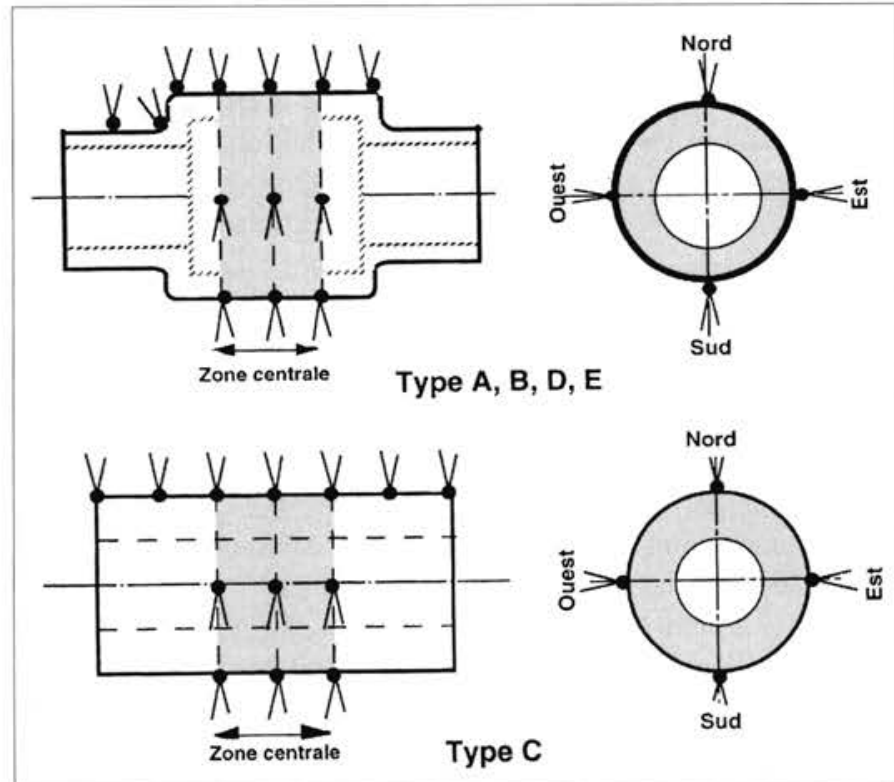

Fig. 2 - Éprouvettes de fatigue thermique cylindriques et creuses et emplacement des thermocouples soudés.

Fig. 2 - Thermal fatigue specimens and location of spot-welded thermocouples.

\begin{tabular}{|c|c|c|c|c|c|c|c|}
\hline \multicolumn{6}{|c|}{ Éprouvette } & \multirow{2}{*}{$\begin{array}{c}\text { Température } \\
\text { maximum } \\
>500^{\circ} \mathrm{C}\end{array}$} & \multirow{2}{*}{$\begin{array}{c}\text { Nombre } \\
\text { de } \\
\text { cycles }\end{array}$} \\
\hline Etat & Type & & $\begin{array}{c}\text { Diamètre } \\
\text { externe }\end{array}$ & $\begin{array}{c}\text { Épais- } \\
\text { seur }\end{array}$ & $\begin{array}{l}\text { Dureté } \\
\text { (HRC) }\end{array}$ & & \\
\hline \multirow{3}{*}{$\begin{array}{l}\text { trempé } \\
\text { et } \\
\text { revenu }\end{array}$} & \multirow{3}{*}{$\begin{array}{l}\text { cylin- } \\
\text { drique }\end{array}$} & A & $D_{1}$ & \multirow{3}{*}{$E P_{1}$} & \multirow{3}{*}{47} & $T_{\operatorname{maxl}}$ & 33000 \\
\hline & & $\mathrm{B}$ & $D_{1}$ & & & \multirow{4}{*}{$\begin{array}{c}T_{\max 2} \\
\left.T_{\max 1}+50^{\circ} \mathrm{C}\right)\end{array}$} & 14000 \\
\hline & & $\mathrm{C}$ & $\mathrm{D}_{2}$ & & & & 6700 \\
\hline \multirow{2}{*}{ recuit } & \multirow{2}{*}{ creuse } & D & \multirow{2}{*}{$D_{1}$} & $E P_{2}$ & \multirow[t]{2}{*}{22} & & 9500 \\
\hline & & $E$ & & $E P_{3}$ & & & 2500 \\
\hline & $D_{1}=0$ & & & & $P_{1}=2$ & $E P_{3}=1,4 \times E$ & \\
\hline
\end{tabular}

Tableau I - Conditions des essais de fatigue thermique.

Table $I$ - Thermal fatigue test conditions.

Dans un premier temps la distribution longitudinale et circonférencielle de température a été mesurée en statique et en dynamique à l'aide de plusieurs thermocouples chromel-alumel (type $\mathrm{K}$ ) de $0,1 \mathrm{~mm}$ de diamètre soudés sur la surface extérieure des éprouvettes témoins (fig. 2). Dans l'état actuel, des gradients dynamiques longitudinaux et circonférenciels de moins de $15^{\circ} \mathrm{C}$ sont obtenus dans la zone centrale des éprouvettes. La période totale d'un cycle thermique (chauffage et refroidissement à l'air comprimé) dont la température maximale dépasse $500^{\circ} \mathrm{C}$, est de l'ordre de quelques dizaines de secondes pour des variations de température de quelques centaines de degrés.

Les surfaces externes des éprouvettes sont polies successivement, d'abord par des papiers abrasifs et ensuite, dans la dernière étape, à la pâte diamantée jusqu'à $1 \mu \mathrm{m}$. L'essai est régulièrement interrompu afin d'observer la surface aux microscopes optique et électronique à balayage. A la fin du test, des coupes circonférencielles et longitudinales ont été réalisées pour les observations. La microdureté Vickers est mesurée sous une charge de $200 \mathrm{~g}$ à froid. Les conditions des essais sont données dans le tableau I.

\section{Résultats et discussions}

La connaissance des sollicitations thermo-mécaniques est nécessaire afin d'analyser l'endommagement des outillages ou des éprouvettes de fatigue thermique. Si une analyse thermoélastique peut s'avérer suffisante dans un premier temps, des calculs plus élaborés sont néanmoins nécessaires car l'amplitude de la déformation plastique cyclique détermine à la fois l'adoucissement cyclique et aussi la durée de vie des aciers martensitiques revenus [11-12].

\section{Analyse thermo-mécanique}

Des calculs thermo-élasto-plastiques ont donc été réalisés en utilisant les données physiques et mécaniques (module d'élasticité, limite d'élasticité, etc.) générées à l'EMAC à différentes températures pour une nuance X $38 \mathrm{CrMoV} 5$ (anciennement Z38CDV5) [11, 19]. Un calcul thermo-élasto-plastique permet de déterminer les contraintes et les déformations d'un cycle stabilisé. Les calculs reportés ici ont été réalisés pour mieux comprendre l'effet de la variation de l'épaisseur sur la génération des déformations et des contraintes thermiques et d'expliquer l'endommagement et l'adoucissement sous sollicitation cyclique anisotherme. Dans l'état actuel, seules les analyses du premier chargement thermo-mécanique sont réalisées et l'adoucissement cyclique n'est pas pris en compte. 


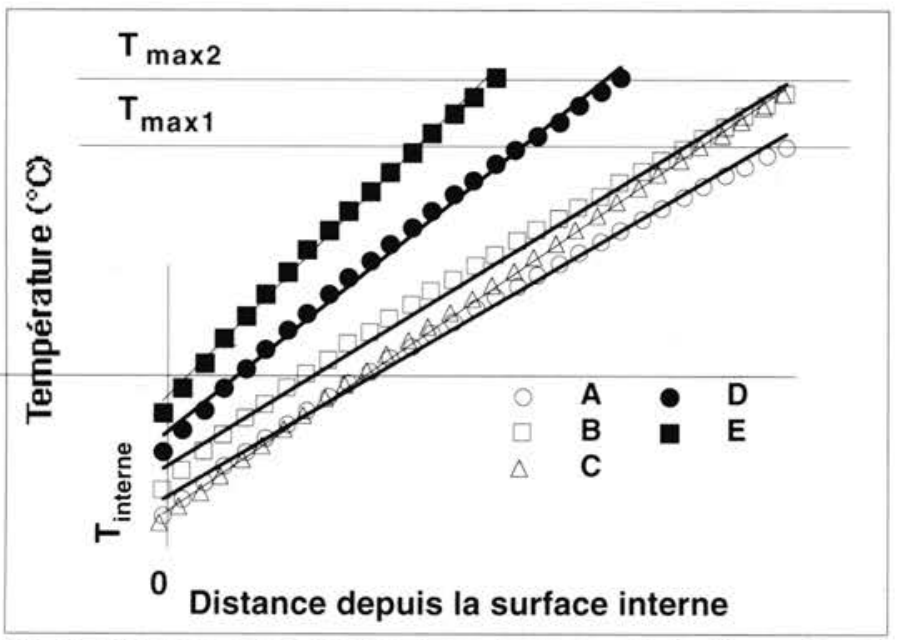

Fig. 3 - Distribution de la température calculée au sein des éprouvettes de fatigue thermique lorsque la température est maximale à la surface externe.

Fig. 3 - Calculated temperature distribution in thermal fatigue specimens when the temperature in thermal cycle is maximum at the external surface.

Le logiciel ABAQuS a été utilisé. Considérant leur symétrie, un quart des éprouvettes a été maillé. Des éléments axisymétriques à huit noeuds, CAX8T ont été utilisés pour les calculs thermiques et mécaniques. Ces éléments sont respectivement bilinéaires et bi-quadratiques pour le calcul thermique et mécanique. Pour le calcul thermique, les températures mesurées à la surface externe ont été imposées aux noeuds des éléments de la surface externe. Pour les éléments de la surface interne (côté eau), le coefficient d'échange et la température de l'eau ont été fixés à $6000 \mathrm{~W} / \mathrm{m}^{2} . \mathrm{K}$ et à $13^{\circ} \mathrm{C}$ respectivement.

En modifiant l'épaisseur et en imposant le même cycle de température-temps à la surface externe, des gradients thermiques différents se créent à l'intérieur de l'éprouvette de fatigue thermique. A titre d'exemple, les températures calculées sont reportées sur la figure 3 en fonction de la distance depuis la surface interne des éprouvettes lorsque la température est maximale à la surface externe. Ces gradients thermiques générent des gradients des déformations mécaniques et des contraintes multiaxiales. Dans la partie centrale de la surface externe des éprouvettes, les gradients longitudinaux sont négligeables. Le rapport des contraintes tangentielles $\left(\sigma_{\theta \theta}\right)$ et longitudinalles $\left(\sigma_{z z}\right)$ qui est proche de 1 (biaxialité) devient supérieur à 1 en dehors de cette zone pour atteindre dans certains cas un rapport de 2 à 2,5 (près de deux extrémités des éprouvettes) [18].

Les calculs thermo-mécaniques montrent que, dans les conditions actuelles, la variation de l'épaisseur de l'éprouvette n'affecte pas d'une manière dramatique la quasi biaxialité de l'état de contrainte dans la partie centrale de la surface externe. L'analyse des composantes de déformations, en revanche, montre que les boucles d'hystéresis de température-déformations mécaniques longitudinales et tangentielles sont du type hors-phase (compression durant le chauffage) et du type enphase (tension au chauffage) en direction radiale.

A titre d'exemple les boucles d'hystéresis de contrainte-déformation mécanique totale (longitudinale et tangentielle) sont représentées sur la figure 4 pour les éprouvettes A, B et C. Les boucles d'hystéresis [18] montrent que l'élévation de la température maximum du cycle thermique ou la modification de la géométrie de l'éprouvette, changent l'amplitude de la déformation mécanique et de la contrainte.

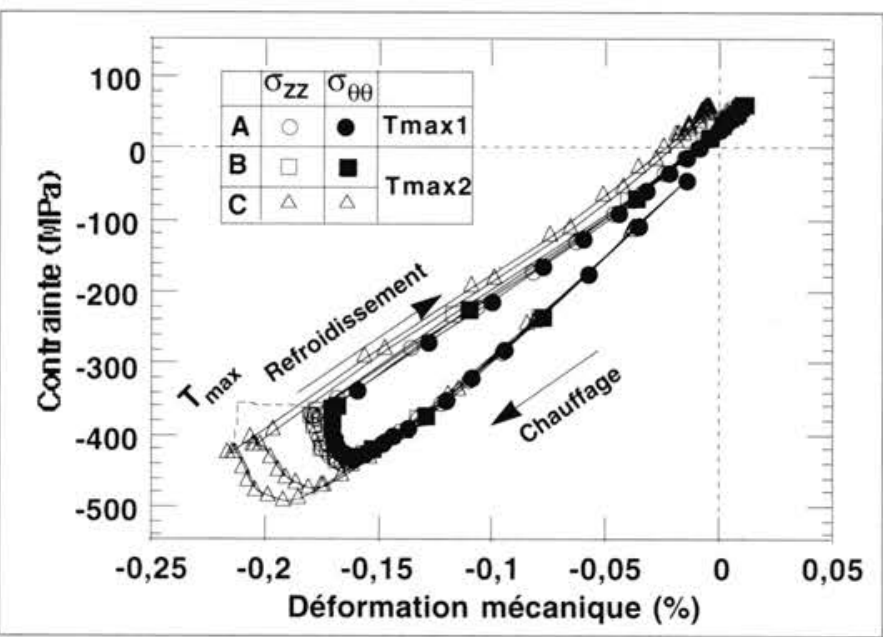

Fig. 4 - Boucles d'hystéresis contrainte-déformation mécanique calculées pour un élément critique à la surface externe des éprouvettes de fatigue thermique $\mathrm{A}$, B et $\mathrm{C}$.

Fig. 4-Calculated thermal stress-mechanical strain hysteresis loops for a critical element at the external surface of specimens $A, B$ and $C$.

\section{Adoucissement}

La perte des caractéristiques mécaniques et la stabilité microstructurale des aciers pour outillage à chaud est généralement déterminée indirectement par la mesure de dureté à froid. Dans la plupart des cas, la diminution de dureté est interprétée comme un indice d'une température de travail élevée (problème de "surchauffe"). En réalité, l'adoucissement d'un acier sollicité cycliquement à chaud, comme par exemple un outillage réel ou des éprouvettes de fatigue anisotherme (fatigue thermique et fatigue thermo-mécanique), n'est pas seulement dû à l'effet combiné de la température et du temps mais aussi à l'action simultanée de l'amplitude de déformation mécanique cyclique, de la température et du nombre de cycles. Négliger ce dernier point peut en effet conduire à des interprétations erronées quant aux causes du périssement des outillages et aussi à une conception et dimensionnement non optimal de l'outillage (température maximum à la surface de travail, systèmes de refroidissement externe et interne, rechargement de l'outillage, etc.). Les activités sur la fatigue isotherme à déformation imposée (fréquence $1 \mathrm{~Hz}$ ) des aciers martensitiques à $5 \%$ de $\mathrm{Cr}$ à l'EMAC [11-12] montrent qu'en effet, ces aciers présentent un adoucissement cyclique. Le degré de l'adoucissement (pour une dureté donnée) dépend à la fois de la température de l'essai et de l'amplitude de la déformation mécanique cyclique.

La figure 5 montre une comparaison entre la dureté mesurée à froid $(200 \mathrm{~g})$ sur des pastilles d'acier Z38CDV 5 traité à $47 \mathrm{HRC}$ qui ont séjourné pendant des temps différents dans un four à $550^{\circ} \mathrm{C}$ sous contrainte nulle [18] et la dureté mesurée post-mortem à froid $(200 \mathrm{~g})$ sur des éprouvettes de fatigue isotherme testées à la même température [20]. Dans les conditions expérimentales reportées ici, un séjour à $550^{\circ} \mathrm{C}$ sous contrainte nulle n'altère quasiment pas la dureté initiale de l'acier, alors qu'un séjour sous une sollicitation cyclique, même à faible amplitude de déformation, peut effectivement abaisser sa dureté à froid. Il est bien évident qu'il n'en va pas de même pour des températures plus élevées [18] où un séjour statique sous contrainte nulle peut aussi faire abaisser la dureté de l'acier.

Les courbes de dureté relative (dureté mesurée à un point donné sur dureté de l'acier à l'état initial) en fonction de la profondeur relative depuis la surface externe (profondeur de point 


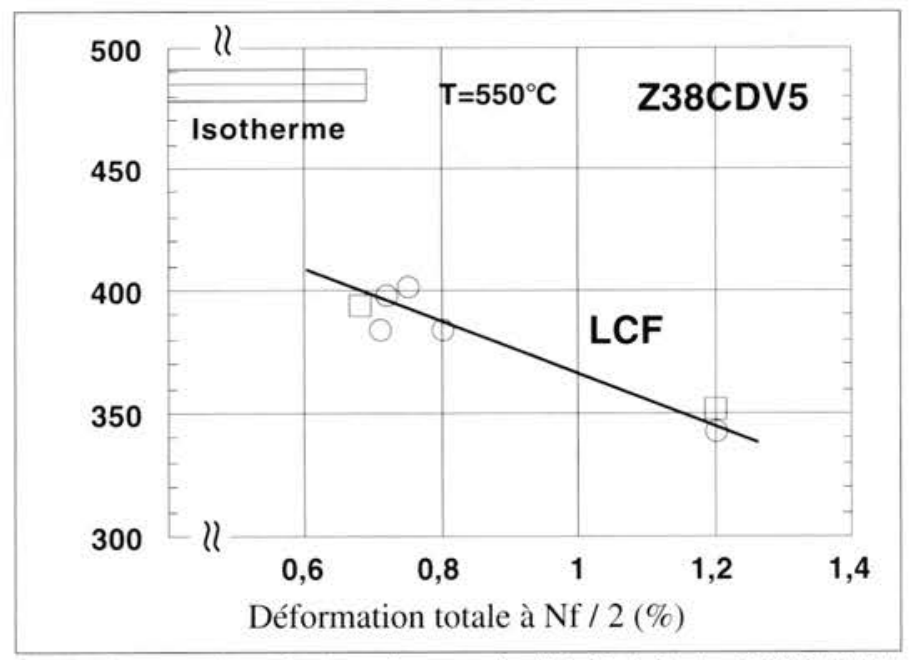

Fig. 5 - Comparaison des duretés mesurées à froid de l'acier X38CrMoV 5 trempé-revenu (47 HRC) après un séjour statique sous contrainte nulle [18] et après des sollicitations en fatigue isotherme [20].

Fig. 5 - Comparison between the hardness of X38 CrMoV 5 steel in as quenched and tempered condition (47 HRC) after static exposures without external loading [18] and after LCF cycling [20].

de mesure sur épaisseur de l'éprouvette) sont reportées sur la figure 6 . La dureté augmente d'une manière quasi linéaire pour atteindre à cœur des éprouvettes la dureté de l'acier à l'état initial. Quelle que soit la condition de sollicitation thermo-mécanique reportée ici, l'acier trempé-revenu à $47 \mathrm{HRC}$ s'adoucit et perd ses caractéristiques mécaniques (abaissement de dureté). En revanche, la dureté de l'acier à l'état recuit reste inchangée.

La diminution linéaire de la dureté depuis la surface externe est, en fait, le résultat de l'effet combiné de "températuredéformation-nombre de cycle" que subit chaque élément. Or, l'analyse numérique a montré [18] que, durant un cycle, des gradients thermiques et des gradients radiaux de déformation mécanique se créent. Par conséquent, pour une géométrie d'éprouvette et un cycle de température-temps imposé à la surface externe, si chaque élément subit le même nombre de cycles, en revanche, son histoire température-déformation mécanique est différente des autres éléments. L'abaissement de la dureté de chaque élément est donc contrôlé particulièrement par son histoire température-déformation mécanique.

Les pertes de dureté des éprouvettes B (environ $40 \%$, à la surface externe, tableau II) et C (environ $50 \%$, à la surface externe, tableau II), dont les diamètres externes sont différents mais qui ont subit le même cycle de température-temps à la surface externe, démontrent le rôle prépondérant de l'amplitude de la déformation mécanique par rapport au nombre de cycle thermique sur l'adoucissement de l'acier. En effet, pour un nombre de cycles thermiques moins élevé (6700 cycles), l'éprouvette C perd davantage sa dureté car elle est sollicitée avec une amplitude de déformation mécanique plus grande.

En revanche, pour les éprouvettes A (environ $25 \%$ de perte de dureté à la surface externe, tableau II) et $\mathrm{B}$ dont la géométrie est la même, mais qui ont été sollicitées à deux $T_{\max }$ différentes en surface externe, il faut prendre en considération la température maximale subie par chaque élément. A titre d'exemple, la figure 4 montre que les amplitudes des déformations mécaniques des éprouvettes $\mathrm{A}$ et $\mathrm{B}$ à la surface externe sont comparables, mais leurs déformations minimales coïncident avec des températures maximales différentes.

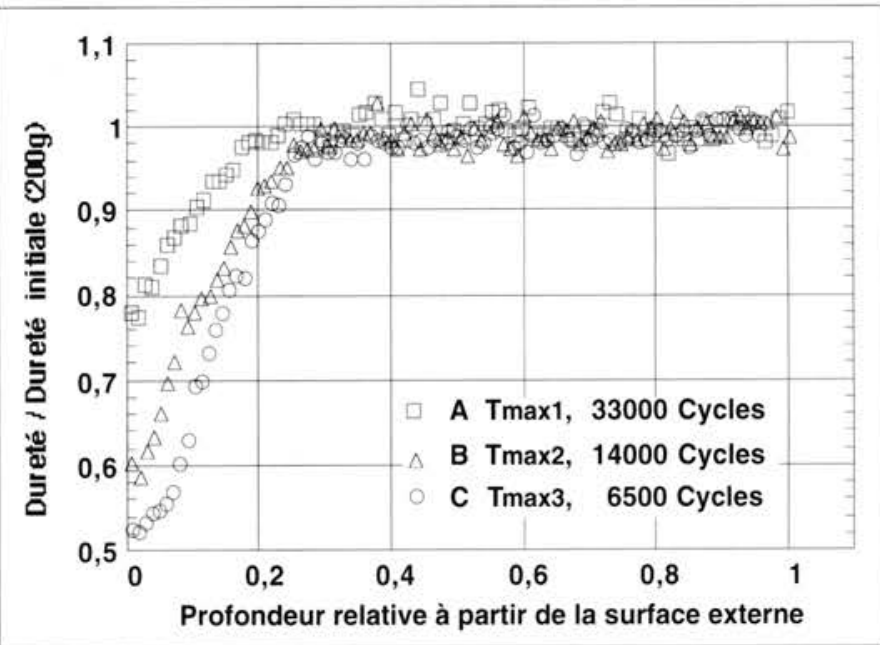

Fig. 6 - Variation de la dureté relative (dureté/dureté initiale de l'acier) des éprouvettes de fatigue thermique $\mathrm{A}, \mathrm{B}$ et $\mathrm{C}$ en fonction de la profondeur relative depuis la surface externe.

Fig. 6 - Variation of the relative hardness (hardness/initial hardness of the steel) as a function of the relative distance from the external surface of the thermal fatigue specimens $A, B$ and $C$.

Tableau II - Conditions de sollicitation, mécanismes d'endommagement et la dureté relative à la surface externe.

Table II - Test conditions, damage mechanisms and relative hardness at the external surface.

\begin{tabular}{|c|c|c|c|c|c|c|}
\hline \multirow{2}{*}{\multicolumn{3}{|c|}{ Éprouvette }} & \multirow{3}{*}{$\begin{array}{c}\text { Température } \\
\text { maximum } \\
T_{\max 1}\end{array}$} & \multirow{3}{*}{$\begin{array}{c}\begin{array}{c}\text { Nombre } \\
\text { de } \\
\text { cycles }\end{array} \\
33000 \\
\end{array}$} & \multicolumn{2}{|c|}{$\begin{array}{c}\text { Surface et } \\
\text { sous-surface externe }\end{array}$} \\
\hline & & & & & $\begin{array}{l}\text { Endomma- } \\
\text { gement }\end{array}$ & $\begin{array}{l}\text { Dureté } \\
\text { relative }\end{array}$ \\
\hline \multirow{3}{*}{$\begin{array}{l}\text { trempé } \\
\text { et } \\
\text { revenu }\end{array}$} & \multirow{3}{*}{$\begin{array}{l}\text { cylin- } \\
\text { drique }\end{array}$} & A & & & \multirow{2}{*}{ Oxydation } & 0,77 \\
\hline & & B & \multirow{4}{*}{$\left(=T_{\max 1}^{\left.T_{\max 2}+50^{\circ} \mathrm{C}\right)}\right.$} & 14000 & & 0,57 \\
\hline & & $\mathrm{C}$ & & 6700 & \multirow{3}{*}{$\begin{array}{c}\text { Faïençage } \\
\text { et } \\
\text { oxydation }\end{array}$} & 0,49 \\
\hline \multirow{2}{*}{ recuit } & \multirow{2}{*}{ creuse } & D & & 9500 & & 1,00 \\
\hline & & $\mathrm{E}$ & & 2500 & & \\
\hline
\end{tabular}

La figure 5 démontre donc l'effet marqué de l'amplitude de la déformation mécanique et de la température sur l'adoucissement cyclique en fatigue anisotherme de l'acier trempé-revenu (47 HRC), comme en fatigue isotherme. Des investigations (mesure de dureté à froid et la mesure de contrainte résiduelle par rayons X) sont en cours pour relier l'abaissement de la dureté en fatigue thermique et les amplitudes des déformations mécaniques calculées à l'intérieur des éprouvettes examinées [18].

\section{Endommagement de la surface externe}

Selon les conditions extrinsèques et intrinsèques de sollicitation $\left(T_{\max }\right.$, type d'éprouvette, et état de traitement thermique de l'acier), deux types d'endommagement ont été identifiés dans la partie centrale des éprouvettes. Dans le cas des éprouvettes A et B, l'oxydation a été le mécanisme unique de l'endommagement. L'élévation de la température, de l'ordre de $50^{\circ} \mathrm{C}$, n'a pas créé le faïençage de l'acier à la surface externe de l'éprouvette $B$. Les couches d'oxydes formées durant la sollicitation thermique s'écaillent et les surfaces nouvellement créées s'oxydent de nouveau. En revanche, lorsque l'amplitude de sollicitation mécanique est élevée (éprouvette C), le faïençage de l'acier est observé dans la zone centrale. Dans cette éprouvette, l'oxydation et l'écaillage de la couche d'oxyde sont aussi observés. 

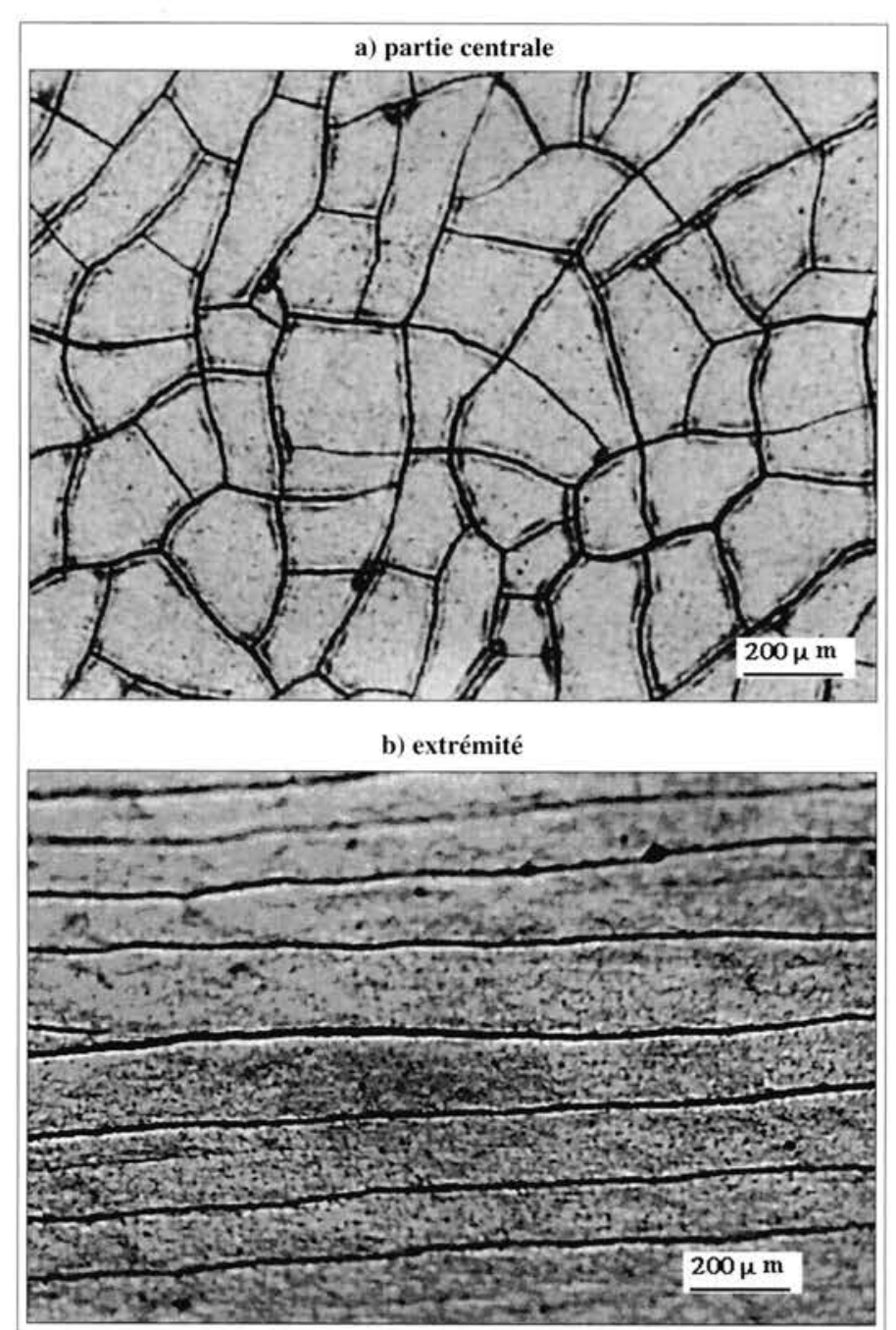

Fig. 7 - Fissurations typiques observées sur la surface externe des éprouvettes de fatigue thermique: (a) biaxiale (faïençage) et (b) uniaxiale.

Fig. 7 - Typical crack initation observed on the external surface of the thermal fatigue specimens: (a) bi-axial (heat checking) and (b) uni-axial.

Dans le cas des éprouvettes D et E (acier à l'état recuit, dont les caractéristiques mécaniques sont moins élevées) testées dans des mêmes conditions que l'éprouvette $C$, le faïençage est de nouveau observé. Pour ces éprouvettes, l'oxydation endommage aussi la surface externe. En augmentant l'épaisseur de l'éprouvette D par rapport à E (diminution du gradient ther- mique), une densité de fissure (cumul de longueur par millimètre carré) moins élevée a été observée après environ 2500 cycles thermiques [18]. Dans le cas où les surfaces sont faïencées, un certain nombre de fissures se propagent à l'intérieur de l'éprouvette. La coalescence de fissure est aussi observée. Le faïençage dans la partie centrale des éprouvettes (l'état de contrainte biaxial) devient progressivement une fissuration plutôt uniaxiale, là où le rapport de contrainte $\sigma_{\theta \theta}$ et $\sigma_{z z}$ devient supérieure à 1 (fig. 7) [18].

\section{Conclusion}

Un banc d'essai de fatigue thermique est développé en utilisant l'induction comme moyen de chauffage. Les essais de fatigue thermique ont été conduits sur l'acier X 38 CrMoV 5 dans un état trempé-revenu à $47 \mathrm{HRC}$ et un état recuit (22 HRC) (paramètres intrinsèques). Le gradient thermique a été modifié à la fois en changeant la géométrie d'éprouvette de fatigue thermique et, aussi, en modifiant la température maximum de cycle thermique (paramètres extrinsèques).

Il a été observé que la surface externe des éprouvettes de fatigue thermique peut se faïencer lorsque l'amplitude de sollicitation thermo-mécanique (température et déformation mécanique) est suffisamment grande. De même, selon les conditions de sollicitation thermo-mécanique, l'endommagement peut se manifester par l'écaillage de la couche d'oxyde sans pour autant qu'il y ait une fissuration dans l'acier. Il a été démontré que sous la sollicitation thermo-mécanique, l'acier trempérevenu ne reste pas stable, et s'adoucit. Le degré de l'adoucissement, mesuré à froid par la dureté, dépend également de l'amplitude de la déformation mécanique et pas uniquement de la durée et la température de l'exposition en température.

\section{Remerciements}

Les auteurs tiennent à remercier la société Aubert et Duval pour l'usinage et les traitements thermiques des éprouvettes de fatigue thermique, M. C. Levaillant, Chef du Centre Matériaux de l'Ecole des Mines pour ses nombreuses contributions et discussions et MM. G. Bernhart et D. Delagnes de l'Ecole des Mines d'Albi-Carmaux pour la mise à disposition de données expérimentales. Les auteurs sont reconnaissants à l'ACRII, Action Concertée de Recherche en Forge, tant pour son soutien financier que pour son autorisation de publication de ces travaux.

\section{RÉFÉRENCES}

[1] E. Glenny, "The Influence of Specimen Geometry on Thermal Fatigue Behaviour", in Thermal and High Strain Fatigue, The Institute of Metals and the Iron and Steel Institute (eds.), London, 1967, pp. 346-363.

[2] F. Rézal-Aria, M. François and L. Rémy, "Thermal Fatigue of MAR-M509 Superalloy - I. The Influence of Specimen Geometry", Fatigue Fract. Engng. Mater. Struct. 11, No. 5, 1998, pp. 277-289.

[3] D. A. WoodFord and D. F. Mowbray, "Effect of Material Characteristics and Test Variables on Thermal Fatigue of Cast Superalloys. A Review", Mater. Sci. and Engg., 1974, pp. 16, 5-43.

[4] L. Northcott and H. G. Baron, "The craze-cracking of metals", J. of the Iron and Steel Institute, december 1956, pp. 385-408.

[5] D. Rousseau, J. P. Riegert, L. Séraphin et R. Tricot, "Fatigue thermique des aciers à outils pour travail à chaud", Colloque sur
«Les Aciers à Outils pour travail à chaud », Saint-Etienne, 2 décembre 1977, pp. 293-321.

[6] P. Skelton, "Crack initiation and growth in simple metal components during thermal cycling, Fatigue at High Temperatures", edited by R.P. Skelton, Elsevier Science Publishers Ltd., New York, 1983, pp. 1-62.

[7] M. Seux, J. C. Saint-Ignan and R. Lévêque, "Improvement of service life from MPM mandrels", Mechanical working and steel processing, 1988, pp. 47-55.

[8] A. Fissolo, B. Marini, A. Berrada, G. Nais and Wident, "Initiation and growth of cracks under thermal fatigue loading for a 316 L type steel", Proceedings of the International Symposium on: Fatigue under Thermal and Mechanical Loading - Mechanisms, Mechanics and Modelling. Petten, The Netherlands, may 22-24, 1995, pp. 67-77. 
[9] F. Meyer-Olbersleben, D. Goldschmidt and F. RéZaï-Aria, "Investigation of the thermal fatigue behaviour of single-crystal nickel-based superalloys SRR99 and CMSX-4", Superalloys 92. $7^{\text {th }}$ Int. Symposium on Superalloys, Seven Springs Mountain Resort, Champion, Pennsylvania, USA, 20-24 sept. 1992, edited by S.D. Antolovich, R.W. Stusrud, R.A. MacKay, D.L. Anton, T. Khan, R.D Kissinger, D.L. Klarstrom, 1992, pp. 785-794.

[10] C. Bournicon, "Sollicitation et modes d'endommagement des outillages de forge à chaud", Traitement Thermique, 246, 1991, pp. 70-77.

[11] D. Delagnes, "Comportement et tenue en fatigue isotherme d'acier à outil Z38CDV5 autour de la transition fatigue oligocyclique-endurance", Thèse de doctorat, Ecole des Mines de Paris, 27 mars 1998.

[12] D. Delagnes, F. Rézaï-Aria, C. Levaillant et A. Grellier, "Comportement et durées de vie en fatigue isotherme de l'acier Z38CDV5", dans ce volume.

[13] H. Samrout, "Loi de comportement élastoviscoplastique à température variable d'un acier de disque de frein pour matériel ferroviaire", Thèse de doctorat, Université de Bordeaux I, $\mathrm{N}^{\circ}$ d'ordre: 1624,6 décembre 1996.

[14] Moulinier, G., "Identification de loi de comportement en fatigue oligocyclique isotherme d'un acier à outil 55NCDV8", Stage d'initiation à la recherche, DEA Génie Mécanique, Université Paul Sabatier (Toulouse III), Ecole des Mines d'Albi-Carmaux, février-juin 1997.

[15] J.L. MALPERTU, L. RÉMY, "Thermomechanical Fatigue Behavior of a Superalloy, Low Cycle Fatigue", ASTM STP 942, H.D. Solomon et al., Philadelphia (1988) pp. 657-671.

[16] C.C. Engler-Pinto Jr, G. Härkegård, B. Ilschner, M.Y. NAZMY, C. Noseda and F. RÉZAÏ-ARIA, "Thermo-mechanical fatigue behaviour of IN738LC", Proceedings of Materials for Advanced Power Engineering 1994, Liège, Belgium, october 3-6 1994, Part-I, pp. 853-862.

[17] B. MrQuel, "Etude du comportement et de l'endommagement en fatigue thermo-mécanique de l'acier F82H-MOD à 9\% de chrome", Stage d'initiation à la recherche, DEA Génie MécaniqueUniversité Paul Sabatier, École des Mines d'Albi-Carmaux, février-juin 1998.

[18] S. Jean et F. Rézaï-Aria, Ecole des Mines d'Albi-Carmaux, travaux non publiés, 1998.

[19] G. Bernhart, Communication privée, Ecole des Mines d'AlbiCarmaux, 1998.

[20] S. Jean, D. Delagnes, C. Levaillant et F. Rézaï-Aria, Ecole des Mines d'Albi-Carmaux, travaux non publiés, 1998. 